\title{
A influência do aleitamento materno para o desenvolvimento da criança
}

\author{
The influence of breastfeeding on child development \\ La influencia de la lactancia materna en el desarrollo infantil
}

Recebido: 23/10/2021 | Revisado: 30/10/2021 |Aceito: 31/10/2021 | Publicado: 01/11/2021

\author{
Glaupe Hadassa Coelho do Nascimento \\ ORCID: https://orcid.org/0000-0002-8117-2853 \\ Centro Universitário Fametro, Brasil \\ E-mail: hadassacoelho18@gmail.com \\ Sara Vieira dos Santos \\ ORCID: https://orcid.org/0000-0003-1756-8367 \\ Centro Universitário Fametro, Brasil \\ E-mail: svs.saravieira@gmail.com \\ Francisca Marta Nascimento de Oliveira Freitas \\ ORCID: https://orcid.org/0000-0002-0044-0925 \\ Centro Universitário Fametro, Brasil \\ E-mail: Francisca.freitas@fametro.edu.br \\ Rosimar Honorato Lobo \\ ORCID: https://orcid.org/0000-0002-8954-2302 \\ Centro Universitário Fametro, Brasil \\ E-mail: rosimar.lobo@ fametro.edu.br
}

\begin{abstract}
Resumo
Introdução: A pesquisa apresenta uma avaliação, com base em estudos bibliográficos, sobre a amamentação e o impacto no desenvolvimento infantil. Objetivo: Verificar a importância do aleitamento materno para o desenvolvimento da criança e as consequências da falta do mesmo. Metodologia: Para a construção do artigo usou-se a revisão da literatura, tendo como base a pesquisa em sites de artigos científicos, exemplo, Scielo, PubMed, Órgãos Governamentais e Revistas, mediante uma pesquisa de informações pertinentes ao tema. Resultado e Discussão: Foram analisadas 50 referências relativas ao tema, os resultados encontrados nos artigos demostraram que bebês amamentados em aleitamento materno exclusivo até os 06 meses de idade tiveram um desenvolvimento melhor do que as amamentadas até os 04 meses ou mesmo as que não amamentaram no primeiro ano de vida. Conclusão: $\mathrm{O}$ aleitamento materno exclusivo é de extrema importância para a mãe e seu bebê, essa conexão de amamentar protege o bebê de infecções respiratórias diminuindo os riscos de alergias, e está relacionado com o melhor desenvolvimento motor da criança, portanto é fundamental que as mães sejam motivadas a amamentarem seus filhos, tendo em vista os benefícios acima mencionados, dentre outro.
\end{abstract}

Palavras-chave: Aleitamento materno; Desenvolvimento da criança; Nutrição materno infantil; Obesidade infantil.

\begin{abstract}
Introduction: The research presents an assessment, based on bibliographic studies, on breastfeeding and its impact on child development. Objective: To verify the importance of breastfeeding for the child's development and the consequences of lack of it. Methodology: For the construction of the article, a literature review was used, based on research on scientific article sites, such as Scielo, PubMed, Government Agencies and Magazines, through a search for information relevant to the topic. Results and Discussion: We analyzed 50 references on the topic, the results found in the articles showed that babies breastfed on exclusive breastfeeding until 06 months of age had a better development than those breastfed until 04 months or even those who did not breastfeed in the first year of life. Conclusion: Exclusive breastfeeding is extremely important for the mother and her baby, this connection of breastfeeding protects the baby from respiratory infections, reducing the risk of allergies, and is related to the better motor development of the child, so it is essential that mothers are motivated to breastfeed their children, in view of the benefits mentioned above, among others.
\end{abstract}

Keywords: Breastfeeding; Child development; Maternal and child nutrition; Child obesity.

\section{Resumen}

Introducción: La investigación presenta una evaluación, basada en estudios bibliográficos, sobre la lactancia materna y su impacto en el desarrollo infantil. Objetivo: Verificar la importancia de la lactancia materna para el desarrollo del niño y las consecuencias de su falta. Metodología: Para la construcción del artículo se utilizó una revisión de la literatura, con base en la investigación en sitios de artículos científicos, como Scielo, PubMed, Agencias Gubernamentales y Revistas, mediante la búsqueda de información relevante al tema. Resultado y Discusión: 
Analizamos 50 referencias sobre el tema, los resultados encontrados en los artículos mostraron que los bebés amamantados en lactancia materna exclusiva hasta los 06 meses de edad tuvieron un mejor desarrollo que los amamantados hasta los 04 meses o incluso los que no amamantaron en el primer mes. año de vida. Conclusión: La lactancia materna exclusiva es sumamente importante para la madre y su bebé, esta conexión de la lactancia materna protege al bebé de las infecciones respiratorias, reduciendo el riesgo de alergias, y se relaciona con el mejor desarrollo motor del niño, por lo que es fundamental que las madres estén motivados a amamantar a sus hijos, en vista de los beneficios antes mencionados, entre otros.

Palabras clave: Amamantamiento; Desarrollo infantil; Nutrición materno-infantil; Obesidad infantil.

\section{Introdução}

A amamentação é a maneira mais eficiente de atender aos aspectos nutricionais, imunológicos, psicológicos e ao desenvolvimento de uma criança nos seus primeiros anos de vida, o leite materno em sua composição contém características bioquímicas ideais para o crescimento e desenvolvimento da criança, sendo benéfico para a mãe e o bebê (Moreira et al., 2021).

De acordo com a Organização Mundial da Saúde (OMS) aleitamento materno é o processo pelo qual o lactente recebe leite materno independente de consumir outros alimentos, sendo o aleitamento materno exclusivo quando o bebê recebe somente o leite da mãe sem incluir outro alimento (Furtado \& Assis, 2012). Estima-se um que em dois copos de 500 ml no segundo ano de vida forneça $95 \%$ das necessidades de vitamina C, $45 \%$ de A, 38\% de proteínas e $31 \%$ do total de energia. (Brasil, 2015).

O leite materno contém 160 substâncias, representado por proteínas, carboidratos, gorduras, vitaminas e células, sendo um alimento essencial para um desenvolvimento satisfatório do bebê, e além de criar um vínculo de interação entre mãe e bebê, proporcionando ganho de peso, e melhor desenvolvimento das estruturas orais envolvidas na ora de sugar, e melhor desenvolvimento cognitivo (Silva et al., 2013). O aleitamento materno ainda traz benefícios de longo prazo para a criança, como maior desempenho escolar, maior quociente de inteligência e maior tempo de estudo (Campos et al., 2020). Os benefícios são de extrema importância, levando a maiores índices de inteligência e acuidade visual, além de proteger contra doenças infecciosas devido às grades quantidades de imunoglobulinas, aumentando a imunidade do bebê (Bruxel \& Sica, 2019). Sendo benéfico não só para o bebê, mas como para mãe, prevenindo de adquirir câncer de mama, câncer de ovários, hemorragia pós-parto, favorecimento do retorno ao peso pré-gestacional mais rápido, além de diminuir o risco de morte por artrite reumatoide (Marques, Cotta \& Priore, 2011). Como é benéfica, a ausência do aleitamento materno também é prejudicial, para o bebê ele irá ter um desenvolvimento mais lento, terá uma imunidade mais baixa, atraso no crescimento, essas são as principais causas.

Sobre o desenvolvimento cognitivo o principal instrumento utilizado no Brasil é a caderneta da criança, toda mãe recebe quando o bebê nasce, para acompanhar o desenvolvimento do seu bebe, são 44 marcos do desenvolvimento infantil de 1 a 36 meses, para a mãe levar ao profissional se o desenvolvimento está ocorrendo bem (Brasil, 2013). Segundo a (Vieira et al., 2015) o desenvolvimento trata-se de uma progressão, por quais todos nós passamos nos primeiros anos de vida, que engloba vários aspectos, físicos, emocionais, cognitivos e sociais.

Nos primeiros anos de vida os neurônios vão se interligando formando uma teia de conexões que permitiram e determinará a capacidade de aprendizagem, memória, raciocínio e habilidades linguísticas. Contudo existem casos em que bebê não tem acesso ao leite materno devido alguns fatores, e devido à ausência do leite ele irá contribuir negativamente no desenvolvimento motor, mastigatório, e consequentemente ao desenvolvimento craniofacial (Braga, Gonçalves \& Augusto, 2020).

Em relação ao autismo em crianças pequenas parece resultado da desmielinização dos neurônios relacionada à oferta inadequada de fator do crescimento semelhante à insulina no recém-nascido, essa deficiência do fator de crescimento 
semelhante à insulina, pode ser devida aos fatores ambientais e genéticos, a amamentação em particular exclusiva tem como aumentar os níveis de fator de crescimento semelhante (IGF), assim compensando uma deficiência congênita do (IGF) (Sociedade Brasileira De Pediatria 2018).

$\mathrm{O}$ aleitamento materno traz vários benefícios para o recém-nascido, além de ser um alimento prático, econômico e sem nenhum custo para a criança, porque é um alimento completo com todas as propriedades nutricionais, vem pronto na temperatura certa. Sendo assim a amamentação pode futuramente proteger contra o excesso de peso e obesidade (Silva, Silva \& Aoyama, 2020).

Este estudo tem por objetivo mostrar a influência do aleitamento materno exclusivo para o crescimento e desenvolvimento da criança, mostrar para as mães que não há outro alimento melhor e nutritivo que substitua o leite materno, sendo ele completo nutricionalmente, prevenindo a criança de ter alergias e infecções respiratórias.

\section{Metodologia}

Para a construção desse artigo científico foi utilizada pesquisa de revisão bibliográfica descritiva da literatura. A revisão descritiva propõe a identificação, registro e análise das características, fatores ou variáveis que se relacionam com o fenômeno ou processo (Perovano, 2014). Dessa forma, embasada em referências bibliográficas e científicas, relatando a influência do aleitamento materno para o desenvolvimento da criança.

Para a coleta de dados foi realizada uma busca nas bases (Scielo, Google acadêmico, Capes, Revistas, Órgãos governamentais, Organização Mundial da Saúde (OMS), Ministério da Saúde (MS). Para a busca dos artigos serão utilizados os descritores: Aleitamento Materno, Desenvolvimento Motor, Nutrição Materno Infantil.

Os critérios de elegibilidade utilizados foram artigos científicos originais, publicações de revistas acadêmicas e diretrizes, abordando a importância do aleitamento materno para um melhor crescimento e desenvolvimento infantil.

Foram utilizados como critérios de inclusão artigos científicos publicados entre os anos de 2011 a 2021 com assuntos relevantes ao tema. Foram excluídos os artigos científicos publicados anteriormente ao ano de 2011 com assuntos que não eram relevantes ao tema.

\section{Resultados e Discussão}

\subsection{Aleitamento materno}

O aleitamento materno é a mais sábia estratégia natural do vínculo mãe e bebê, que traz proteção e nutrição para a criança, o leite ele reúne as características ideais na quantidade certa de nutrientes, além de promover vantagens na diminuição de mortalidade infantil. (Maciel et al., 2013).

Segundo a pesquisa realizada, pode - se constatar diversas vantagens em decorrência do aleitamento, dentre as principais, destacam-se o fato de que a criança amamentada ao seio estará protegida contra alergias e infecções, fortalecendose com os anticorpos da mãe e evitando diarreias, pneumonias, otites e meningites. Levando em conta que, além disso, a aleitação favorece o desenvolvimento dos ossos e fortalece os músculos da face, facilitando também a fala, regulando a respiração e prevenindo os problemas na dentição. Assim como o leite traz benefícios, a ausência dele nos primeiros anos de vida trará malefícios para a criança e afetará no seu desenvolvimento cognitivo, a criança terá desnutrição, ficar com deficiências de nutrientes, terá um desenvolvimento mais lento, e pode ter infecção causada pelas fórmulas infantis desnecessárias nos primeiros anos de vida (Bezerra et al., 2017).

Ter como incentivar e promover a aleitamento materno, as iniciativas são logo após o parto, quando o período de reatividade do recém-nascido está presente, fortalecendo o vínculo entre mãe e filho, levando a redução da mortalidade 
neonatal e materna, garantindo a diminuição de hemorragia e favorecendo a involução uterina, sendo melhor todo esse aconchego, amor e união em seu lar. (Aparecida et al., 2014).

\subsection{Composição do leite materno}

O leite materno tem tudo que o bebê precisa para seu desenvolvimento, crescimento e saúde, tais como nutrientes, fatores imunológicos, tróficos, possuem bactérias importantes para a modulação da microbiota intestinal, por isso é importante lembrar que qualquer alimento sem ser o leite introduzido antes do tempo irá fazer mal ao bebê, por sua microbiota ainda está em desenvolvimento. (Oliveira et al., 2020).

As vitaminas A, C, D, E, K e complexo B que são componentes do leite materno têm grandes funções como, crescimento, formação óssea, formação do sistema imunológico, desenvolvimento das funções neurológicas entre outras, ele contém também vitaminas antioxidantes. (Saqueti et al., 2019).

O leite humano é um alimento completo, contendo até água, fatores de proteção, vitaminas, proteínas, nutrientes, entre outros que são de suma importância nos primeiros meses de vida da criança, não de outro leite, o seu leite é poderoso e seu neném não precisa de outro alimento sem ser o leite materno. (Mascarenhas et al., 2015).

Quadro 1: Composição do leite materno.

\begin{tabular}{|c|l|}
\hline Água & $\begin{array}{l}\text { A água é o maior componente do leite e desempenha papel fundamental na regulação da } \\
\text { temperatura corporal. Na água estão dissolvidas ou suspensas as proteínas, os compostos } \\
\text { nitrogenados não proteicos, os carboidratos, os minerais (íons monovalentes) e as } \\
\text { vitaminas hidrossolúveis (C e Complexo B). }\end{array}$ \\
\hline Proteínas & $\begin{array}{l}\text { Na primeira semana o leite humano, colostro, é rico em proteínas protetoras } \\
\text { especialmente a imunoglobulina secretória A, que age contra infecções e alergia } \\
\text { alimentar. O leite maduro contém mais proteínas nutritivas que o colostro. }\end{array}$ \\
\hline Lipídios & $\begin{array}{l}\text { O leite humano disponibiliza quantidades adequadas de lipídios, que aumentam com o } \\
\text { tempo de lactação e são compostos principalmente por triglicerídeos, que fornecem cerca } \\
\text { de 50\% da energia do leite. }\end{array}$ \\
\hline Carboidratos & $\begin{array}{l}\text { A lactose é o carboidrato mais abundante no leite humano. Este carboidrato favorece a } \\
\text { absorção do cálcio e fornece galactose para a mielinização do sistema nervoso central, } \\
\text { além de energia. }\end{array}$ \\
\hline Vitaminas e Minerais & $\begin{array}{l}\text { O leite humano fornece todas as vitaminas e minerais, micronutrientes necessários para o } \\
\text { crescimento e desenvolvimento infantil. Durante os primeiros seis meses o aleitamento } \\
\text { materno exclusivo garante boa biodisponibilidade de todos os nutrientes. }\end{array}$ \\
\hline
\end{tabular}

Fonte: Adaptada de Caputo Neto (2013).

\subsection{Benefícios do leite materno para mãe e o bebê}

O leite materno para o bebê agrega além de benefício calórico e proteico, a proteção imunitária necessária, no primeiro ano de vida da criança, o leite traz benefícios na área cognitiva, motora, e ainda aumento dos indicadores gerais da saúde dos lactentes, prevenindo as crianças de terem alguma doença futura. (Bezerra et al., 2012).

$\mathrm{O}$ ato de amamentar estimula o vínculo afetivo entre mãe e bebê. A união física e emocional gera sentimentos agradáveis, que gera amor, carinho, e apego entre ambos que é fundamental para o desenvolvimento infantil, e se pode considerar que a falta dele trará prejuízos à criança. (Oliveira et al., 2016).

Amamentar traz benefícios para a mãe como, involução uterina, sangramento reduzido, infecção reduzida, adiposidade reduzida e peso, estresse e ansiedade reduzidos, e longo prazo reduzindo o câncer de mama, ovário e diabetes. (Ciampo \& Ciampo, 2018).

No ato da sucção o bebê está fazendo exercício físico contínuo, que propicia o desenvolvimento da musculatura facial, isso faz com que ele tenha uma respiração correta, esse é um dos benefícios de amamentar. (Martins \& Santana 2013). 
Quadro 2: Os benefícios da amamentação tanto para a mãe e para o bebê.

\begin{tabular}{|c|l|}
\hline Para Mãe & $\begin{array}{l}\text { Diminui o sangramento no pós-parto; } \\
\text { Acelera a perda de peso; reduz a incidência de câncer de mama, ovário e } \\
\text { endométrio; Evita a osteoporose; protege contra doenças cardiovasculares, como o } \\
\text { infarto. }\end{array}$ \\
\hline Para o Bebê & $\begin{array}{l}\text { Maior contato com a mãe; Melhora a digestão e minimiza as cólicas; desenvolve a } \\
\text { inteligência quanto maior o tempo de amamentação; reduz o risco de doenças } \\
\text { alérgicas; diminui as chances de desenvolver doença de Crohn e linfoma; estimula e } \\
\text { fortalece a arcada dentária; previne contra doenças contagiosas, como a diarreia. }\end{array}$ \\
\hline
\end{tabular}

Fonte: Adaptada de Caputo Neto (2013).

\subsection{Ausência do leite materno e as consequências para a saúde}

O pouco de informação ou conhecimento pode fazer com que a mãe deixe de amamentar seu bebê, por mitos ou crenças acerca da amamentação, fazendo a mãe introduzir outros alimentos que são maléficos para a saúde do bebê, e a ausência do leite nos primeiros anos de vida vão trazer prejuízos assim com traz benefícios para o bebê e a mãe, exemplo riscos de mortalidade, gastroenterite aguda, otite, infecção respiratória baixa, doenças inflamatórias do intestino, previne o bebê de ter alergias entre outras, e as mães os riscos de câncer de mama, câncer do ovário, depressão pós-parto. (Tenório, Mello \& Oliveira, 2018).

A substituição do leite materno por leite artificial faz com que ocorra um aumento de mortes de crianças, em torno de 1,5 milhões a cada ano, no mundo, a maioria é por diarreia, por não ter um saneamento bom de água, a higiene é precária fazendo o preparo do leite com a água contaminada, tem uma solução para os números diminuírem é apenas o incentivo ao aleitamento materno exclusivo. (Silva et al., 2016).

As fases iniciais da vida são de extrema importância para o desenvolvimento humano, as quais sofrem fatores de influências nutricionais e metabólicas. Com isso a introdução de outros alimentos na dieta do lactente é uma fase muito crítica pela alta susceptibilidade de levar a acriança ao déficit nutricional e ao desenvolvimento de doenças, e consigo as alergias alimentares a mais comum é a proteína do leite da vaca. (Silva et al., 2019).

Estima-se que as alergias alimentares vêm tendo um aumento nas últimas décadas, sendo que a prevalência é $6 \%$ a $8 \%$ afetando as crianças menores de três anos em decorrência a exposição mais precoce e inadequada de alimentos na dieta das crianças, substituindo o leite materno rico em todos os nutrientes pelo leite da vaca, com isso desencadeando alergias alimentares. (José et al.,2016).

A otite média é uma inflamação do ouvido médio de início rápido, causada principalmente por vírus ou bactérias, acredita-se que a incidência de otite média nos lactentes esteja relacionada com o aleitamento materno artificial, porque o leite artificial não contem as substâncias, o nutriente imunológico que contém no leite materno, causando assim doenças e infecções pelo manuseio e preparação incorreta desses leites artificiais que não tem benefício nenhum para o bebê. (Nadal et al., 2017).

\subsection{Crescimento e desenvolvimento infantis}

O crescimento saudável é alcançado com uma adequada alimentação, sendo o leite materno fundamental na primeira fase da vida, visto que ele reúne todos componentes nutricionais ideais com balanceamento adequado de nutrientes, ressaltando que nos primeiros dois anos de vida ocorrem à fase de crescimento rápido, sendo o período mais vulnerável aos distúrbios do crescimento (Santos, Bispo \& Cruz, 2016).

O leite materno exclusivo contém vários benefícios para o bebê, contém nutrientes que são essenciais para o crescimento e desenvolvimento infantil, rico em vitaminas, carboidratos, proteínas, gorduras, sais minerais e água (Amaral et al., 2015). O sistema nervoso central começa a se desenvolver-se na vida intrauterina do bebê, e continua até por volta dos 5 
anos da criança, o leite é imprescindível, o encéfalo é 50\% formado por esse nutriente (Nascimento et al., 2017). O leite materno tona-se essencial para a formação, o ácido araquidônico (AA) e o ácido Docosa-Hexaenoico são componentes lipídicos importantes para o desenvolvimento das membranas celulares, células da retina e do sistema nervoso (Sociedade Brasileira De Pediatria, 2013). Com a sucção correta exercida durante a amamentação irá contribuir para um desenvolvimento estomatognático saudável (Cassimiro et al., 2019). Substâncias encontradas no leite materno fresco são essenciais para o máximo neurodesenvolvimento infantil (Eidelman., 2013).

Segundo (Silva et al., 2016) o leite materno possui todos os nutrientes que o bebê necessita para o seu crescimento e desenvolvimento saudável, proporcionando um bom desenvolvimento para a criança, protegendo-a de infecções gastrointestinais, doenças respiratórias, desnutrição, doenças crônicas, as crianças amamentadas tem um crescimento e desenvolvimento melhor do que as que não foram amamentadas.

Estudos demostram também uma associação benéfica entre o aleitamento materno e um melhor desenvolvimento não só na infância, mas também na adolescência, devido os nutrientes compostos no leite que são essenciais para o desenvolvimento cerebral, tanto na ação bioquímica e funcional (Oliveira et al., 2017). Crianças amamentadas com o leite materno exclusivo tem menos alergias, infecções, diarreias, doenças respiratórias e otites, e menos chance de apresentar obesidade e diabetes tipo 2, possuem também melhor desempenho nos testes de inteligência (Brasil, 2020).

$\mathrm{O}$ amamentar é uma função por excelência da mulher e, de acordo com as expectativas culturais, constitui-se momento de realização plena da feminilidade, de satisfação pessoal, mesmo sob a influência do meio social (Cabral et al., 2013).

Venancio, Saldiva e Monteiro (2013) afirmam que existem tendencia gradativa da amamentação no Brasil, todavia apontam importantes desafios para acelerarem o ritmo de crescimento dessa prática, rumo ao alcance das recomendações. Por meio de tal cenário o profissional de saúde tem um desafio de passar a informação para as mães, isso implica não somente a necessidade de conhecimentos técnicos, mas principalmente conhecimentos, habilidades e atitudes para acolher dúvidas, preocupações que as mães passem (Brasil, 2015).

A Política Nacional de Aleitamento Materno recomenda que o país progrida com estratégias para a promoção, proteção e apoio ao aleitamento materno, visando a melhoria dos indicadores na saúde da criança (Vargas et al., 2016).

\subsection{Obesidade infantil}

De acordo com o Ministério da Saúde (2021) a obesidade infantil é resultado de uma variedade de fatores genéticos, comportamentais, que atuam em vários contextos: familiar, escolar, social. Fatores que influenciam ainda na gestação como o excesso de peso da mãe, a inadequada alimentação na gestação, um aleitamento de curta duração. Uma das causas da obesidade infantil é a oferta de alimentos excessivamente calóricos e de baixo valor nutritivo a criança frágil de idade (Wannmacher, 2016). O acréscimo da obesidade infantil se torna acima de tudo preocupante porque a possibilidades da criança sobrepeso transforma-se em um adulto sobrepeso é grande (Weffort, 2019). Além do mais crianças obesas apontam marcadores precoces de doenças cardiovascular, aumento do risco de fraturas, dificuldade respiratória e resistência à insulina (Barroso \& Souza 2020).

Ademais a composição do leite materno é completamente diferente dos outros leites contendo todas as propriedades nutricionais que uma criança necessita, os demais leites contêm uma quantidade maior de calorias fazendo com que as crianças se tornem mais propensas a obesidade infantil (Bastos et al., 2020). estudos mostram indícios sobre a proteção da amamentação contra várias doenças como diarreia, otite media aguda, doenças infecciosas, reduz a morbidade e a mortalidade neonatal, e tem um efeito protetor contra o sobrepeso e obesidade infantil (Contarato et al., 2016). 
Ter uma alimentação adequada propõe um crescimento e desenvolvimento adequado, evitando déficits nutricionais e previne o indivíduo de adquirir uma hipertensão arterial, diabetes tipo 2, doenças cardiovascular, osteoporose e obesidade (Macedo et al., 2020). O momento de introdução e a quantidade de alimentos, incluindo alimentos sólidos inseridos na dieta das crianças no princípio da vida podem levar a um acréscimo do risco de manifestar obesidade precocemente e as comorbidades a ela associadas (Nascimento et al., 2016).

por essa razão é de extrema importância escolher os alimentos adequados na hora de fazer uma introdução alimentar nas crianças.

De acordo com (Bellodi, 2018) tais experimentos e pesquisas com animais insinua que as primeiras experiencias nutritivas do ser vivo podem estabelecer a sua vulnerabilidade a doenças crônicas na fase adulta, assim como a obesidade.

Fatores no início da vida que auxiliam o progresso da obesidade infantil: peso materno, diabetes gestacional, alimentação com formulas variadas, introdução precoce de alimentos sólidos (Pereyra et al., 2021). Aleitamento materno exclusivo AME para bebes até os seis meses e o prosseguimento do aleitamento materno AM com uma alimentação adequada até os 2 anos de idade, aleitamento materno exclusivo quando a criança recebe o leite direto da mama de sua mãe sem introdução de outros líquidos e alimentos (Moraes et al., 2021). Segundo (Souza, Molero \& Gonçalves 2021) a introdução de frutas, cereais, legumes, verduras, raízes, ovos e carnes são as medidas mais saudáveis para promover a saúde da criança.

\section{Conclusão}

Crianças amamentadas até os seis meses com o leite materno exclusivo tiveram um melhor crescimento e desenvolvimento cognitivo em relação as crianças que não foram amamentadas até os seis meses no aleitamento materno exclusivo tiveram um crescimento e desenvolvimento mais lento.

O leite é um alimento adequado para o crescimento e desenvolvimento do bebe nos seis meses de vida, sem a necessidade de complementação, basta dar apoio a mãe quanto a amamentação e orientar e incentivar o aleitamento materno sobre os benefícios para não ocorrer o desmame precoce ou uma introdução alimentar antes do tempo.

Portanto, é importante incentivar o aleitamento materno para promoção de crescimento e desenvolvimento adequados das crianças e prevenção de doenças infecciosas, gastrointestinais e carências nutricionais.

\section{Referências}

Amaral, L. J. X. et al. (2015). Fatores que influenciam na interrupção do aleitamento materno exclusivo em nutrizes. Rev. Gaúcha de Enfermagem, Paraíba, 36. https://doi.org/10.1590/1983-1447.2015.esp.56676.

Aparecida, K., et al. (2014). Percepção das mães em relação ao aleitamento materno no período pós-parto. ABCS Health SCI, 39(3), 147. https://www.portalnepas.org.br/abcshs/article/view/648/647.

Barroso, M., \& Souza, A. (2020). Obesidade, sobrepeso, adiposidade corporal e risco cardiovascular em crianças e adolescentes. Arq Bras Cardiol. https://www.scielo.br/j/abc/a/WGqvnyMQ7LShPbY4j9mHsvL/?lang=pt.

Bastos, C. et al. Deficiência do aleitamento materno exclusivo como contribuinte para a obesidade infantil. Revista Eletrônica Acervo Cientifico, 17. 2020. https://acervomais.com.br/index.php/cientifico/article/download/5757/3587/.

Bellodi, A.M. (2018). Obesidade em Crianças e Adolescentes: temperamento, estresse, coping e risco psicossocial familiar. 221p Tese (Programa de PósGraduação em Psicologia) - Pontifícia Universidade Católica de Campinas, SP. http://tede.bibliotecadigital.puccampinas.edu.br:8080/jspui/bitstream/tede/1054/2/ANITA COLLETES BELLODI.pdf.

Bezerra, J., et al. (2017). Hábitos modernos relacionados a amamentação. Rev. baiana enferm. https://periodicos.ufba.br/index.php/e nfermagem/article/view/1824.

Bezerra, V., et al. (2012). Aleitamento materno exclusivo e fatores associados à sua interrupção precoce: estudo comparativo entre 1999 e 2008. Rev.paul. pediatr. São Paulo, 30(2). https://www.scielo.br/scielo.php?pid=S0103 05822012000200004\&script=sci_abstract\&tlng=pt.

Braga, M. S., Gonçalves, M. S., \& Augusto, C. R. (2020). Os Benefícios do aleitamento materno para o desenvolvimento infantil. 6(9). www.brazilianjournals.com/index.php/BRJD/article/view/16985/15832. 
Brasil. (2015). Ministério da Saúde, caderno de atenção básica: saúde da criança, aleitamento materno e alimentação complementar. (2a ed.), 15. https://bvsms.saude.gov.br/bvs/publicacoes/saude_crianca_aleitamento_materno_cab23.pdf.

Brasil. (2013). Ministério da Saúde, Secretaria de Atenção à Saúde: caderneta de saúde da criança: passaporte para a cidadania. Brasília. 2013. https://bvsms.saude.gov.br/bvs/publicacoes/caderneta_saude_crianca_menino.pdf

Brasil. (2015). Ministério da Saúde, Secretaria de Atenção à Saúde: Estratégia Nacional para Promoção do Aleitamento Materno e Alimentação Complementar Saudável no Sistema Único de Saúde: Manual de Implementação. Brasília. https://bvsms.saude.gov.br/bvs/publicacoes/estrategia _nacional_promocao_aleitamento_materno.pdf.

Brasil. (2020). Ministério da Saúde, Secretaria de Atenção Primária a Saúde (SAPS): Pesquisa Inédita revela que Índices de Amamentação Cresceram no Brasil. Brasília. https://aps.saude.gov.br/noticia/9416.

Brasil. (2021). Ministério Da Saúde: Obesidade Infantil afeta 3,1 milhões de crianças menores de 10 anos no Brasil. https://www.gov.br/saude/ptbr/assuntos/noticias/obesidade-infantil-afeta-3-1-milhoes-de-criancas-menores-de-10-anos-no-brasil.

Bruxel, R., \& Sica, C. (2019). Analise de proteína e micronutrientes em amostra de leite humano. Revista Brasileira de Obesidade, nutrição e emagrecimento. São Paulo. 13(78), ISSN 1981-9919. 2019. http://www.rbone.com.br/index.php/rbone/article/view/909/662.

Cabral, P., et al. (2013). Motivos do sucesso da amamentação exclusiva na perspectiva dos pais. Revista eletrônica de enfermagem, Goiânia, Brasil, 15(2). https://www.revistas.ufg.br/fen/article/view/16996/14824.

Campos, P., et al. (2020). Contato pele a pele e aleitamento materno de recém-nascido em um hospital universitário. Revista Gaúcha de Enfermagem. https://doi.org/10.1590/1983-1447.2020.20190154. Acesso em 30 de setembro 2021.

Cassimiro, I., et al. (2019). A importância da amamentação natural para o sistema estomatognático. Revista Uningá, 56(55). 55. <http://revista.uninga.br/index.php/uninga/article/view/2678/1995.

Ciampo, L., \& Ciampo, I. (2018). Aleitamento materno e os benefícios do aleitamento materno para a saúde da mulher. Rev. Bras. Ginecol. Obstet., 40(6), 355. https://www.scielo.br/pdf/rbgo/v40n6/0100-7203-rbgo-40-06-00354.pdf.

Contarato, F., et al. (2016). Efeito independentemente do tipo de aleitamento no risco de excesso de peso e obesidade em crianças entre $12-24$ meses de idade. Cadernos de saúde pública. https://doi.org/10.1590/0102-311X00119015.

Eidelman, A. I. (2013). Amamentação e desenvolvimento cognitivo: existe uma associação? Jornal de Pediatria, 4, 89. https://www.scielo.br/j/jped/a/BMJ WjQ5r43hKrNsdtWHktgp/?lang=pt\&format=pdf.

Furtado, L., \& Assis, T. (2012). Diferentes Fatores que Influenciam na Decisão e na Duração do Aleitamento Materno: uma revisão da literatura. Movimenta, 5(4),304. https://www.revista.ueg.br/index.php/movimenta/article/view/7073/4842.

José, D., et al. (2016). Relação entre desmame e alergias alimentares. 17(3), 67-68. https://revistas.ufpr.br/academica/article/download/49129/30211.

Macedo, R., et al. (2020). Associação entre aleitamento materno e excesso de peso em pré-escolares. Acta Paul Enferm. https://doi.org/10.37689/actaape/2020AO0025.

Maciel, A., et al. (2013). "Conhecimento De Gestantes E Lactantes Sobre Aleitamento Materno Exclusivo." Revista Brasileira em Promoção da Saúde, 26(3), 312. https://www.redalyc.org/articulo.oa?id=40829885002.

Marques, S., Cotta, M., \& Priore, E. (2011). Mitos e Crenças sobre o aleitamento materno. Departamento de nutrição e saúde, universidade federal de viçosa. Minas gerais. https://doi.org/10.1590/S1413-81232011000500015.

Martins, M., \& Santana, L. (2013). Benefícios da amamentação para saúde materna. Interfaces cientificas- saúde e ambiente. 1(3), 90. https://periodicos.set.edu.br/saude/article/download/763/443/0.

Mascarenhas, A., et al. A (2015). percepção das puérperas frente à atuação do enfermeiro na promoção do aleitamento materno em um Hospital amigo da Criança do Estado do Pará. Rev. para. med., 29(3). http://files.bvs.br/upload/S/0101-5907/2015/v29n3/a5558.pdf.

Moraes, et al. (2021). Amamentação nos seis primeiros meses de vida de bebes atendidos por consultoria em lactação. Revista latino am. Enfermagem. https://www.scielo.br/j/rlae/a/5CS4DJJb7J8j3mPSQHMMFWR/?lang=pt\&format=pdf. <http://dx.doi.org/10.1590/1518-8345.3538.3412 >

Moreira, et al. (2021). Os benefícios da amamentação exclusiva na vida e saúde das crianças e sua genitora. V colóquio estadual de pesquisa multidisciplinar III congresso nacional de pesquisa multidisciplinar e II de empreendedorismo. <https://publicacoes.unifimes.edu.br/ind ex.php/coloquio/article/view/1016/958>.

Nadal, F., et al. (2017). Investigação das práticas maternas sobre aleitamento materno e sua relação com a infecção de vias aéreas superiores e otite média. Rev. CEFAC, São Paulo, 19(3), 388. https://www.scielo.br/scielo.php?pid=S1516-18462017000300387\&script=sci_abstract\&tlng=pt.

Nascimento, V., et al. (2016). Aleitamento materno, introdução precoce de leite não materno e excesso de peso na idade pré-escolar. Revista Paulista de Pediatria. https://doi.org/10.1016/j.rppede.2016.05.002.

Nascimento, J., et al. (2017). Influência do aleitamento materno no desenvolvimento do sistema nervoso. Revista de trabalhos acadêmicos universo recife. 4 , 2-1. http://revista.universo.edu.br/index.php?journal=1UNICARECIFE2\&page=article\&op=view\&path\%5B\%5D=4940\&path\%5B $\% 5 \mathrm{D}=3018$.

Oliveira, E., et al. (2020). O excesso de peso modifica a composição nutricional do leite materno? Uma revisão sistemática. Cienc. Saúde coletiva, Rio de janeiro, 25(10). https://www.scielosp.org/pdf/csc/2020.v25n10/3969-3980/pt. 
Oliveira, I., et al. (2016). Saberes Maternos Sobre a Relação entre Amamentação Natural e Hábitos Bucais deletérios. Off Health Science, Universidade Federal do Piauí, 18(2), 75-79. https://docs.bvsalud.org/biblioref/2017/06/833880/01-saberes-maternos.pdf.

Oliveira, S. et al, (2017). Associação entre o Aleitamento Materno, Introdução Alimentar e Desenvolvimento Neuropsicomotor nos Primeiros seis meses de Vida. Distúrbios da Comunicação, São Paulo, 29(2). https://revistas.pucsp.br/index.php/dic/article/view/29637/22997.

Pereyra, I., et al. (2021). Peso ao nascer, ganho ponderal e obesidade em crianças no Uruguai: estudo prospectivo desde o nascimento. Revista Paulista de Pediatria, 39. https://doi.org/10.1590/1984-0462/2021/39/2019088>.

Perovano, D. G. (2014). Manual de Metodologia Científica. Editora Juruá.

Santos, O., Bispo, B., \& Cruz, D. (2016). Padrão de Aleitamento e Estado Nutricional de Crianças até seis meses de idade. Hu Revista Juiz de Fora, 42(2). 120. <https://periodicos.ufjf.br/index.php/hurevista/article/view/2514>

Saqueti, B. et al. Revisão sobre as vitaminas presentes no leite materno. Encontro internacional de produção cientifica. 2019 http://rdu.unicesumar.edu.br/handle/123456789/3985.

Silva, E., et al. (2016). Benefícios do aleitamento materno no crescimento e desenvolvimento infantil: uma revisão sistemática. Revista das Ciências da saúde do Oeste Baiano- Higia. Bahia, 1(2), 151. http://fasb.edu.br/revista/index.php/higia/article/view/151.

Silva, E., et al. (2016). Benefícios do aleitamento materno no crescimento e desenvolvimento infantil: uma revisão sistemática. Revista das ciências da saúde do oeste baiano. 1(2), 150. http://www.fasb.edu.br/revista/index.php/higia/article/download/125/131.

Silva, M., et al. (2019). A introdução alimentar precoce e o risco de alergias: Revisão da literatura. Revista eletrônica de enfermaria, 54, 486, https://scielo.isciii.es/pdf/eg/v18n54/pt_1695-6141-eg-18-54-470.pdf.

Silva, O., et al. (2013). Benefícios provenientes do aleitamento materno exclusivo. Revista Uningá Review, 16(2). http://revista.uninga.br/index.php/uningareviews/article/view/1473.

Sociedade Brasileira de Pediatria. (2018). Departamento cientifico de aleitamento materno, amamentação: A Base da Vida. 6. www.sbp.com.br/fileadmin/user_upload/_21162c-DC_-_Amamentaçao_-_da_vida.pdf.

Sociedade Brasileira de Pediatria. (2013). Impacto da amamentação no quociente de inteligência de crianças de oito anos de idade. 38, edição 4 , https://doi.org/10.1016/j.jped.2012.12.010.

Souza, B., Molero, M., \& Gonçalves, R. (2023). Alimentação complementar e obesidade infantil. Revista multidisciplinar da saúde (RMS), 3(2), 3 https://revistas.anchieta.br/index.php/RevistaMultiSaude/article/view/1724/1534

Tenorio, M., Mello, C., \& Oliveira, A. (2018). Fatores associados à ausência de aleitamento materno na alta hospitalar em uma maternidade pública de Maceió, Alagoas, Brasil. Ciênc. saúde coletiva, 23(11), 3550. https://www.scielo.br/pdf/csc/v23n11/1413-8123-csc-23-11-3547.pdf acesso 10/.

Vargas, A. et al. (2016). Atuação dos Profissionais de Saúde da Estratégia Saúde da Família: Promoção da Pratica do Aleitamento Materno. Revista Baiana de Enfermagem, salvador, 30(2), 3. http://biblioteca.cofen.gov.br/wp-content/uploads/2017/06/Atua\%C3\%A7\%C3\%A3o-dos-profissionais-de-sa\%C3\%BAde-daestrat $\% \mathrm{C} 3 \%$ A 9 fia-sa\%C3\%BAde-da-fam\%C3\%ADlia-promo\%C3\%A7\%C3\%A3o-da-pr\%C3\%A1tica-do-aleitamento.

Venancio, S., Saldiva, S., \& Monteiro, C. (2013). Tendencia secular da amamentação no Brasil. Revista Saúde Pública. 47 (6), 1208. https://doi.org/10.1590/S0034-8910.2013047004676.

Vieira, A., et al. (2017). As influencias do aleitamento materno para o desenvolvimento infantil: uma revisão integrativa de literatura. Revista Espacios 38(12). www.revistaespacios.com/a17v38n12p15.pdf.

Wannmacher, L. (2016). Obesidade como um fator de risco para morbidade e mortalidade: Evidencias sobre o Manejo com Medidas não Medicamentosas. OPAS/OMS. 1(7). https://www.paho.org/bra/dmdocuments/Fasciculo 7.pdf.

Weffort, V. (2019). Sociedade Brasileira de Pediatria- Departamento de Nutrologia. Obesidade na infância e adolescência: manual de orientação. (3a ed.), Manual de Orientação. https://www.sbp.com.br/fileadmin/user_upload/Manual_de_Obesidade_-_3a_Ed_web_compressed.pdf. 